\title{
Erratum to: Plasma level of lipopolysaccharide-binding protein is indicative of acute graft-versus-host disease following allogeneic hematopoietic stem cell transplantation
}

Haige Ye $\cdot$ Meng Lv $\cdot$ Xiaosu Zhao

Xiangyu Zhao $\cdot$ Xiaojun Huang

Published online: 20 July 2012

(C) The Japanese Society of Hematology 2012

Erratum to: Int J Hematol (2012) 95:680-688

DOI 10.1007/s12185-012-1076-z

The authors' affiliations appeared incorrectly in the article cited above. The correct authors' affiliations are as follows.

The online version of the original article can be found under doi:10.1007/s12185-012-1076-z.

H. Ye $\cdot$ M. Lv $\cdot$ X. Zhao $\cdot$ X. Zhao $\cdot$ X. Huang $(\bowtie)$

Peking University People's Hospital,

Peking University Institute of Hematology,

Beijing Key Laboratory of Hematopoietic Stem Cell

Transplantation, No 11 Xizhimen South Street,

Beijing 100044, China

e-mail: xjhrm@medmail.com.cn

H. Ye

Department of Hematology, The First Affiliated Hospital

of Wenzhou Medical College, Wenzhou, Zhejiang, China 